\title{
Níveis de metionina + cistina total para frangos de corte de 22 a 42 dias de idade mantidos em ambiente termoneutro ${ }^{1}$
}

\author{
Adhemar Rodrigues de Oliveira Neto일 Rita Flávia Miranda de Oliveira ${ }^{3}$, Juarez Lopes Donzele ${ }^{3}$, \\ Sergio Luiz de Toledo Barreto ${ }^{3}$, Roberta Gomes Marçal Vieira Vaz ${ }^{4}$, Eliane Gasparino ${ }^{5}$ \\ 1 Parte da tese de Doutorado do primeiro autor. Projeto financiado pela FAPEMIG. \\ 2 Nutricionista - Nutrição DSM Produtos Nutricionais do Brasil Ltda. \\ ${ }^{3}$ DZO/UFV. \\ ${ }^{4}$ Doutoranda do DZO/UFV \\ 5 DZO/UEM.
}

RESUMO - O experimento foi realizado para determinar a exigência de metionina + cistina (met + cis) para frangos de corte dos 22 aos 42 dias de idade mantidos em ambiente termoneutro $\left(23,5^{\circ} \mathrm{C}\right)$. Foram utilizados 240 frangos de corte machos Avian Farms, com peso médio inicial de 827 g, em delineamento inteiramente casualizado, com cinco níveis de met + cist $(0,659 ; 0,704 ; 0,750 ; 0,796$ e $0,841 \%)$, seis repetições e oito aves por unidade experimental. $\mathrm{O}$ aumento do nível de met + cis influenciou positivamente a conversão alimentar (CA), o consumo de met + cis, o peso absoluto da coxa e o peso relativo da sobrecoxa. Não foi verificado efeito dos níveis de met + cis sobre o peso final, o ganho de peso, o consumo de ração, os pesos absoluto e relativo do peito, da carcaça e da gordura abdominal, o peso absoluto da sobrecoxa e o peso relativo da coxa, assim como para os pesos absolutos e relativos dos órgãos (coração, fígado, moela, proventrículo, pulmão e intestinos). O nível de $0,727 \%$ de met + cis total $(0,661 \%$ met + cis digestível), correspondente à relação met + cis/lis total de $70 \%(72 \%$ para lis digestível), proporcionou melhor conversão alimentar em frangos de corte mantidos em ambiente termoneutro.

Palavras-chave: ambiente térmico, aminoácidos sulfurosos, desempenho, exigências nutricionais, frango de corte

\section{Levels of total methionine + cystine for 22-to-42-day-old broilers kept under thermoneutral environment}

\begin{abstract}
The experiment was carried out to determine the requirement of methionine + cystine (met + cys) for broilers from 22-to-42-day-old kept under thermoneutral environment $\left(23.5^{\circ} \mathrm{C}\right)$. A total of 240 Avian Farm male broilers, with $827 \mathrm{~g}$ average initial weight were distributed in a randomized experimental design with five levels of met + cys $(0.659$, $0.704,0.750,0.796$, and $0.841 \%$ ), six replications and eight birds per experimental unit. The increase of met + cys level influenced positively feed: gain ratio, met + cys intake, drumstick absolute weigh and thigh relative weight. No effect of met + cys levels was observed on the final weight, weight gain, feed intake, breast, carcass and abdominal fat absolute and relative weight, thigh absolute weight, and drumstick relative weight, or for relative and absolute weight of the organs (heart, liver, gizzard, proventriculus, lung and intestines). The level $0.727 \%$ met + total cys $(0.661 \%$ digestible met + cys), corresponding to the ratio met + cys/total lys of $70 \%$ ( $72 \%$ for digestible lys) gave the best feed: gain ratio for male broilers kept in thermoneutral environment.
\end{abstract}

Key Words: broiler, nutritional requirement performance, sulphur amino acids, thermal environment

\section{Introdução}

Além da fundamental contribuição do melhoramento genético, o sucesso da avicultura de corte decorre, em grande parte, da nutrição. Nas últimas décadas, o ganho de peso médio diário aumentou de 20 para mais de 50 g/dia e a idade de abate reduziu de 12 para seis semanas (Silva, 2005). Essa precocidade das linhagens modernas tem demandado constantes pesquisas sobre a real exigência de aminoácidos, principalmente os sulfurosos.
Para se obter ótimo desempenho das aves, além da preocupação com o ambiente térmico, é necessário que a proteína da dieta forneça níveis suficientes de aminoácidos essenciais e não-essenciais para permitir máxima síntese protéica e atender às exigências de outros processos metabólicos (Fancher \& Jensen, 1989). É ainda recomendável manter uma relação adequada entre os aminoácidos da dieta para que sejam evitadas perdas energéticas decorrentes do desequilíbrio entre os aminoácidos, uma vez que, quando em excesso, os 
aminoácidos serão eliminados, exigindo gasto de energia no processo.

O desequilíbrio aminoacídico em aves, provocado pelo excesso ou pela deficiência de aminoácidos, pode causar diminuição no ganho de peso, no consumo de ração e na retenção de energia (Kino \& Okumura, 1986), podendo ocasionar também acúmulo de gordura no fígado, aumento no catabolismo do aminoácido limitante e, em alguns casos, levar a deficiência de vitaminas.

Em dietas práticas para frangos de corte, formuladas à base de milho e farelo de soja, a metionina é o primeiro aminoácido limitante e seus níveis na dieta podem ser influenciados pelos níveis de lisina, colina e arginina. Além disso, esse aminoácido pode funcionar como doador de grupamentos metil, necessários para síntese de carnitina e creatina (Schutte et al., 1997).

Algumas pesquisas têm sido realizadas com vistas ao estabelecimento das necessidades de metionina das aves, no entanto, tem havido ainda considerável divergência quanto aos fatores que podem influenciar a exigência desse aminoácido.

O NRC (1994) preconiza valores de metionina+cistina total para frangos de corte de $0,90 \%$ para a fase de 1 a 21 ; 0,72\% para 22 a 42 ; e $0,60 \%$ para 43 a 56 dias de idade para dietas contendo $3.200 \mathrm{kcal} / \mathrm{kg}$ de energia metabolizável. Entretanto, deve-se considerar que o frango utilizado antes de 1994 era geneticamente diferente das linhagens atuais. Rostagno et al. (2000) recomendaram valores de metionina+cistina total de $0,90 \%$ para a fase de 1 a $21 ; 0,83 \%$ para 22 a 42; e 74\% para 43 a 56 dias de idade.

As linhagens comerciais de frango de corte atuais são resultado do sucesso de programas de seleção para rápido crescimento e conformação corporal, favorecendo especialmente a musculatura peitoral (Scheuermann et al., 2003) e, com o aumento na comercialização do frango em partes, em detrimento da carcaça inteira, têm sido demandadas linhagens comerciais com maior porcentagem de coxa e peito e menor teor de gordura na carcaça. Por essa razão, a indústria avícola tem estado constantemente interessada em avaliar o desempenho de linhagens comercialmente disponíveis, considerando o peso e o rendimento de carne de peito como as mais importantes características, e alguns estudos têm demonstrado estreita relação entre o nível de metionina da dieta e o rendimento da musculatura peitoral em frangos de corte (Huyghebaert et al., 1994; Schutte \& Pack, 1995).

Assim, torna-se evidente a necessidade contínua de se estabelecerem as exigências nutricionais para frangos de corte para utilização adequada dos nutrientes da dieta pelas aves, principalmente nas fases de crescimento e terminação, de modo que essas aves possam atingir sua máxima eficiência produtiva.

\section{Material e Métodos}

O experimento foi conduzido em câmaras climatizadas no Laboratório de Bioclimatologia Animal do Departamento de Zootecnia do Centro de Ciências Agrárias da Universidade Federal de Viçosa, no município de Viçosa, MG.

Foram utilizados 240 frangos de corte machos da linhagem Avian Farms no período dos 22 aos 42 dias de idade, com peso inicial de $827 \pm 2,5 \mathrm{~g}$. O delineamento experimental utilizado foi o inteiramente casualizado, com cinco tratamentos (níveis de met + cis totais), seis repetições e oito aves por unidade experimental.

Durante o período inicial ( 1 a 21 dias de idade), as aves foram criadas em galpão convencional, sob manejo e alimentação tradicionais, como descrito por Gomes et al. (1996). Completados os 22 dias de idade, os frangos foram pesados e transferidos para as câmaras climáticas, quando se deu início ao período experimental. As aves foram mantidas no experimento até os 42 dias de idade.

As aves foram alojadas, em grupos de oito, em compartimento de baterias metálicas $(0,85 \times 0,85 \mathrm{~m})$ com piso telado de área igual a $0,72 \mathrm{~m}^{2}$, dotado de comedouros e de bebedouros tipo calha, constituindo-se cada gaiola uma unidade experimental. O programa de luz adotado durante todo o período experimental foi o contínuo, com 24 horas de luz artificial, por meio de duas lâmpadas fluorescentes de 75 watts por sala.

O monitoramento da temperatura e da umidade de cada sala foi feito por meio de termômetros (de máxima e mínima, de bulbo seco e bulbo úmido e de globo negro) colocados à altura intermediária em relação ao compartimento central da bateria. As leituras dos termômetros foram realizadas diariamente durante todo o período experimental, três vezes ao dia (8, 13 e 18 h). Posteriormente, os dados foram convertidos no índice de temperatura de globo e umidade (ITGU), segundo Buffington et al. (1981).

A ração experimental (basal) foi formulada à base de milho, farelo de soja e glúten de milho e suplementada com minerais e vitaminas (Tabela 1) para atender às exigências nutricionais, segundo Rostagno et al. (2000), exceto de metionina + cistina digestível. Os níveis de met + cis das dietas $(0,045 ; 0,091 ; 0,137 ; 0,182)$ foram obtidos em substituição ao amido de milho e resultaram em rações isoprotéicas e isocalóricas com 0,704; 0,750; 0,796 e 0,841\% de met + cis total em relação à ração basal. As exigências nutricionais mínimas seguiram recomendações de Rostagno et al. (2000), com exceção do nível de metionina + cistina digestível.

Os níveis de met + cis totais avaliados neste estudo corresponderam aos níveis de met + cis digestíveis calculados 
Tabela 1 - Composição centesimal da ração experimental Table 1 - Percentage composition of the experimental diet

\begin{tabular}{lr}
$\begin{array}{l}\text { Ingrediente } \\
\text { Ingredient }\end{array}$ & $(\%)$ \\
\hline Milho (8,72\% PB ${ }^{1}$ ) (Corn, 8.72\% CP) & 64,500 \\
Farelo de soja (46,72\% PB ${ }^{1}$ ) (Soybean meal, 46.72\% CP ${ }^{1}$ ) & 29,120 \\
Amido (Starch) & 0,335 \\
Óleo vegetal (Vegetable oil) & 2,390 \\
Fosfato bicálcico (Dicalcium phosphate) & 1,493 \\
Calcário (Limestone) & 1,191 \\
Sal (Salt) & 0,402 \\
Mistura mineral ${ }^{2}$ (Mineral mix) & 0,050 \\
Mistura vitamínica ${ }^{3}$ (Vitamin mix) & 0,100 \\
DL-metionina (99\%) (DL-methionine) & 0,150 \\
L-treonina (L-threonine) & 0,017 \\
L-triptofano (L-tryptophan) & 0,012 \\
Cloreto de colina (60\%) (Choline cloride) & 0,125 \\
Anticoccidiano ${ }^{4}$ (Anticoccidian) & 0,050 \\
Bacitracina de zinco (Zinc bacitracin) & 0,055 \\
BHT & 0,010
\end{tabular}

Composição calculada

Calculated composition

PB (CP) $(\%)$

EM (ME) $(\mathrm{kcal} / \mathrm{kg})$

19,18

3.099

Metionina total (Total met) (\%)

Metionina digestível (Digestible met) (\%)

0,376

0,358

Met + cis total (Total met + cys) $(\%)$

0,659

Met + cis digestível (Digestible met + cys) $(\%)$

0,593

Lisina total (Total lysine) (\%)

1,039

Lisina digestível (Digestible lysine) (\%)

0,912

${ }^{1}$ Valor obtido no laboratório de Nutrição Animal do DZO/UFV, de acordo com metodologia descrita por Silva \& Queiroz (2002) (Value obtained in the Laboratory of Animal Nutrition of DZO/UFV, according to the metodology described by Silva \& Queiroz, 2002).

2 Quantidade/kg do produto (content/kg of the product): Fe - $100 \mathrm{mg}$; Co - 2,0 mg: $\mathrm{Cu}$ - 20,0 mg; Mg - 160,0 mg; Zn - 100,0 mg; I - 2,0 mg; q.s.p. - 1.000 g.

3 Quantidade/kg do produto (content/kg of the product): vit. A - 10.000 U.I.; vit. $\mathrm{D}_{3}-2.000$ U.I.; vit. E - 30 U.I.; vit. $B_{1}-2,0$ mg; vit. $B_{2}-6,0$ mg; vit. $B_{6}$ - 4,0 mg; vit. $B_{12}-0,015$ mg; ác. pantotênico (pantothenic acid) - 12,0 mg; biotina (biotine) - 0,1 mg; vit. $\mathrm{K}_{3}-3,0 \mathrm{mg}$; ác. fólico (folic acid) - 1,0 mg; ác. nicotínico (nicotinic acid) - 50,0 mg; Se - 0,25 mg; q.s.p. - 1.000 g.

${ }^{4}$ Coxistac $12 \%$ - Salinomicina (Salinomicine).

de 0,$593 ; 0,638 ; 0,684 ; 0,730$ e $0,775 \%$ e às relações entre met + cis digestível/lisina digestível de 65, 70, 75, 80 e 85\%. O fornecimento de ração e água foi ad libitum, sendo a água trocada duas vezes ao dia. O consumo foi calculado pela diferença entre a quantidade de ração fornecida, os desperdícios e as sobras das rações experimentais, pesadas aos 22 e 42 dias de idade.

As variáveis estudadas foram: consumo de ração, ganho de peso, conversão alimentar, consumo de met + cis, deposição de gordura e de proteína na carcaça, rendimento de carcaça e pesos relativo e absoluto dos cortes nobres e da carcaça, das vísceras e dos órgãos comestíveis (coração, fígado e moela) e não-comestíveis (proventrículo, pulmão e intestino).

Ao final do experimento ( $42^{\circ} \underline{-}$ dia), as aves foram pesadas após jejum alimentar de 6 horas. Quatro aves de cada unidade experimental, considerando o peso médio de cada unidade experimental (10\% acima e abaixo da média), foram abatidas por meio de deslocamento cervical. Após serem sangradas, depenadas e evisceradas, as aves foram pesadas. O rendimento de carcaça, expresso em porcentagem, foi obtido pela razão entre o peso da carcaça (eviscerada, com pés e cabeça) e o peso de jejum.

Para os cortes nobres (peito com osso, coxa e sobrecoxa), procedeu-se à pesagem e ao cálculo dos rendimentos em relação ao peso da carcaça eviscerada. As carcaças inteiras (incluindo pés e cabeça), sem as vísceras e o sangue, foram moídas, durante 15 minutos, em cutter comercial de 30 HP e 1.775 rpm, obtendo-se amostra para posterior procedimento laboratorial.

As amostras de carcaça foram inicialmente pré-secas em estufa com ventilação forçada a $\pm 60^{\circ} \mathrm{C}$, durante 72 horas. Posteriormente, em razão do alto teor de gordura, foi realizado pré-desengorduramento pelo método a quente, em aparelho extrator do tipo "Soxhlet”, durante 4 horas. Após esta etapa, as amostras foram moídas e acondicionadas em vidros para posteriores análises.

Os teores de água e gordura extraídos no preparo das amostras foram considerados para correção dos valores das análises. As análises bromatológicas (EE, PB e MS) das carcaças dos frangos de corte foram realizadas no Laboratório de Nutrição Animal do Departamento de Zootecnia da UFV, conforme metodologia descrita por Silva (1990).

Um grupo adicional de 22 aves, com 22 dias de idade, foi abatido para determinação da composição corporal das aves no início do experimento. As deposições de gordura e proteína nas carcaças foram calculadas comparando-se as composições das carcaças das aves no início (22 dias de idade) e no final (42 dias de idade) do período experimental.

Os órgãos e as vísceras foram cortados longitudinalmente e pendurados à sombra para que o sangue escorresse, sendo pesados em seguida. O intestino foi aberto em toda sua extensão para retirada de seu conteúdo. Em seguida, foi lavado e, depois de escorrido o excesso de água, foi pesado. O peso relativo (\%) das vísceras e dos órgãos foi calculado em relação ao peso da carcaça eviscerada.

As análises das características de desempenho, de carcaça, de cortes nobres e de peso das vísceras foram realizadas utilizando-se o programa SAEG (Sistema para Análise Estatística e Genética) desenvolvido pela Universidade Federal de Viçosa (1992). As estimativas de exigência de metionina + cistina foram estabelecidas por meio de modelos de regressão polinomial e do modelo descontínuo - LRP (Linear Response Plateau) conforme o melhor ajuste dos dados. 


\section{Resultados e Discussão}

Constam na Tabela 2 os valores médios de temperatura e de umidade relativa do ar no interior das câmaras climáticas verificados durante o período experimental, assim como o valor médio do ITGU, calculado segundo Buffington et al. (1981).

Não foi observado efeito $(\mathrm{P}>0,10)$ do nível de met + cis digestível da ração sobre o ganho de peso dos frangos de corte no período de 22 a 42 dias de idade (Tabela 3). Assim, pode-se inferir que o nível de $0,659 \%$ de met + cis atendeu às exigências das aves mantidas em ambiente termoneutro. Resultado semelhante foi obtido por Mitchell \& Robbins (1984), que, avaliando a exigência de aminoácidos sulfurados para frangos de corte dos 28 aos 42 dias de idade, não verificaram variação no ganho de peso das aves quando o

Tabela 2 - Condições ambientais médias observadas durante o período experimental nas câmaras climáticas para frangos de corte de 22 a 42 dias de idade mantidos em ambiente termoneutro

Table 2 - Environmental average conditions observed during the experimental period in the climatic chambers for the male broilers from 22 to 42 days kept under thermoneutral environment

\begin{tabular}{lc}
\hline $\begin{array}{l}\text { Variável } \\
\text { Varialble }\end{array}$ & $\begin{array}{c}\text { Valor } \\
\text { Value }\end{array}$ \\
\hline $\begin{array}{l}\text { Temperatura do ar média }\left({ }^{\circ} \mathrm{C}\right) \\
\text { Average air temperature }\end{array}$ & $23,5 \pm 0,5$ \\
$\begin{array}{l}\text { Umidade relativa média }(\%) \\
\text { Average relative humidity }(\%)\end{array}$ & $67,3 \pm 3,1$ \\
$\begin{array}{l}\text { Temperatura de globo negro média }\left({ }^{\circ} \mathrm{C}\right) \\
\text { Average black globe temperature }\left({ }^{\circ} \mathrm{C}\right)\end{array}$ & $23,5 \pm 0,5$ \\
$\begin{array}{l}\text { Índice de temperatura de globo e umidade (ITGU) } \\
\text { Globe humidity temperature index }(\mathrm{BGHI})\end{array}$ & $70,7 \pm 0,7$ \\
\hline
\end{tabular}

nível de metionina + cistina total da ração aumentou de 0,705 para 0,945\%. Corroborando ainda esses resultados, Graber et al. (1971) concluíram que a exigência de metionina + cistina total de frangos de corte com cinco semanas de idade não foi superior a $0,630 \%$.

O resultado deste estudo também está consistente com o obtido por Silva et al. (1997), que verificaram que o nível de $0,653 \%$ de metionina + cistina total proporcionou a melhor resposta de ganho de peso das aves.

Em contrapartida, o resultado de ganho de peso obtido neste estudo diverge dos observados por Jensen et al. (1989), Rodrigueiro et al. (2000) e Albino et al. (1999), que constataram efeito significativo dos níveis de metionina + cistina total da ração sobre o ganho de peso de frangos de corte dos 22 aos 42 dias e destacaram que as melhores respostas foram obtidas com níveis de metionina + cistina superiores a $0,75 \%$.

O consumo de ração não foi influenciado pelo aumento do nível de metionina + cistina da ração. Esse resultado foi similar aos obtidos por Mitchell \& Robbins (1984) e Silva et al. (1997), que também não constataram influência do nível dos aminoácidos sulfurados da ração sobre o consumo de ração de frangos na fase de crescimento. Entretanto, os dados de consumo de ração observados nesse estudo diferem daqueles relatados por Sekis et al. (1975), Wheeler \& Latshan (1981) e Rodrigueiro et al. (2000), que observaram variação significativa no consumo, em razão do nível de metionina + cistina da ração.

A variação de resultados entre os trabalhos pode estar relacionada às diferenças no nível de proteína e no perfil aminoacídico das rações experimentais, entre outros fatores, como genética, sexo e ambiente. De acordo com Tafuri et al. (1984), o grau de desbalanço (excesso ou deficiência) de

Tabela 3 - Desempenho de frangos de corte aos 42 dias de idade mantidos em ambiente termoneutro alimentados com rações com diferentes níveis de metionina + cistina

Table 3 - Performance of male broiler at 42 days of age kept under thermoneutral environment fed diets with different levels of methionine + cystine

\begin{tabular}{|c|c|c|c|c|c|c|}
\hline \multirow[t]{2}{*}{$\begin{array}{l}\text { Variável } \\
\text { Variable }\end{array}$} & \multicolumn{5}{|c|}{$\begin{array}{c}\text { Nível de met }+ \text { cis } \\
\text { Met }+ \text { cys level }(\%)\end{array}$} & \multirow[t]{2}{*}{ CV (\%) } \\
\hline & 0,659 & 0,704 & 0,750 & 0,796 & 0,841 & \\
\hline Peso final (g) (Final weight) & 2.441 & 2.458 & 2.455 & 2.434 & 2.460 & 1,95 \\
\hline Ganho médio diário (g) (Average daily gain) & 1.613 & 1.631 & 1.629 & 1.607 & 1.633 & 2,94 \\
\hline Consumo de ração (g) (Feed intake) & 3.046 & 3.060 & 3.033 & 3.050 & 3.123 & 3,20 \\
\hline Conversão alimentar ${ }^{1}$ (Feed:gain ratio) & 1,89 & 1,88 & 1,86 & 1,90 & 1,91 & 1,82 \\
\hline Consumo met + cis total $(\mathrm{g})^{2}$ (Methionine + cystine intake) & 20,1 & 21,5 & 22,7 & 24,3 & 26,3 & 3,32 \\
\hline
\end{tabular}

\footnotetext{
${ }^{1}$ Efeito quadrático $(P<0,09)$ (Quadratic effect, 0.09).
}

${ }^{2}$ Efeito linear $(P<0,01)$ (Linear effect, $\left.P<0.01\right)$. 
metionina determina o padrão de resposta do consumo de ração das aves.

O nível de metionina + cistina influenciou $(\mathrm{P}<0,09)$ de forma quadrática a conversão alimentar dos frangos, que melhorou até o nível estimado de $0,727 \%$ de metionina + cistina total (Figura 1), correspondente a $0,661 \%$ de met + cis digestível. Efeito quadrático dos níveis de aminoácidos sulfurados sobre a conversão alimentar de frangos de corte dos 21 aos 42 dias de idade também foi observado por Schutte \& Pack (1995a), Silva et al. (1997), Rodrigueiro et al. (2000) e Albino et al. (1999). Apesar da similaridade da resposta de conversão entre os trabalhos, o nível de metionina + cistina que proporcionou a melhor resposta neste estudo $(0,727 \%)$ foi menor que os níveis determinados por esses autores, que corresponderam a valores superiores a $0,880 \%$. No entanto, Baker et al. (1996) observaram melhor resposta

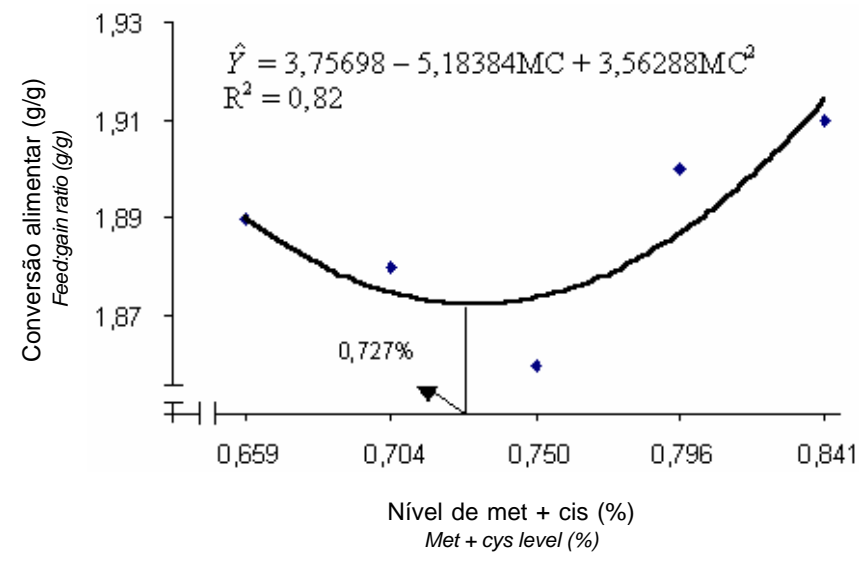

Figura 1 - Conversão alimentar de frangos de corte aos 42 dias de idade mantidos em ambiente termoneutro alimentados com rações com diferentes níveis de metionina + cistina

Figure 1 - Feed:gain ratio of male broilers at 42 days of age, kept under thermoneutral environment fed diets with different levels of methionine + cystine. de conversão alimentar das aves no nível de 0,70\% de metionina + cistina.

O nível de proteína utilizado nas rações experimentais pode ter contribuído para a variação de resultados entre os trabalhos. Determinando a exigência de metionina + cistina para frangos de corte dos 22 aos 42 dias de idade, Silva et al. (1997) verificaram melhor resposta de desempenho no nível de $0,826 \%$ quando utilizaram ração com $17,5 \%$ de PB e com o nível de 0,910\% quando o nível de PB da ração correspondeu a $20 \%$.

O consumo de metionina + cistina aumentou $(\mathrm{P}<0,01)$ de forma linear conforme elevaram seus níveis na ração (Tabela 5). Uma vez que o consumo de ração dos frangos não foi influenciado $(\mathrm{P}>0,10)$ pelos níveis de metionina + cistina, a variação no consumo desses aminoácidos esteve diretamente relacionada às suas concentrações nas rações. Considerando os resultados de desempenho, determinou-se que a melhor resposta foi obtida no consumo estimado de 22,2 g de metionina + cistina no período avaliado.

Com exceção do peso relativo de sobrecoxa $(\mathrm{P}<0,02)$, que variou de forma quadrática (Tabela 5), o peso relativo dos demais cortes e da gordura abdominal não foram influenciados $(\mathrm{P}>0,10)$ pelo nível de metionina + cistina da ração (Tabela 4).

Os resultados de peso relativo da gordura abdominal foram semelhantes aos verificados por Silva et al. (1997) e Jensen et al. (1989), que, avaliando níveis de metionina + cistina para frangos de corte, também não verificaram variação significativa no rendimento de gordura abdominal das aves aos 42 dias de idade.

Rodrigueiro et al. (2000) constataram redução linear no rendimento de gordura abdominal de frangos de corte machos aos 42 dias de idade conforme aumentaram o nível de metionina+cistina da ração $(0,630$ a 0,930\%). De forma semelhante, Schutte \& Pack (1995) também verificaram

Tabela 4 - Pesos relativos dos cortes e da gordura abdominal de frangos de corte aos 42 dias de idade mantidos em ambiente termoneutro alimentados com rações com diferentes níveis de metionina + cistina

Table 4 - Relative weights of cuts and abdominal fat of male broilers at 42 days of age kept under thermoneutral environment fed diets with different levels of methionine + cystine

\begin{tabular}{lrrrrrr}
\hline $\begin{array}{l}\text { Variável } \\
\text { Variable }\end{array}$ & \multicolumn{3}{c}{$\begin{array}{c}\text { Nível de met + cis } \\
\text { Met + cys level (\%) }\end{array}$} \\
\cline { 2 - 5 } & 0,659 & 0,704 & 0,750 & 0,796 & 0,841 \\
\hline Peito com osso (Breast with bone) & 28,8 & 28,4 & 28,2 & 29,0 & 28,8 & 4,83 \\
Coxa (Thigh) & 12,6 & 12,7 & 12,8 & 13,0 & 12,6 & 4,29 \\
Sobrecoxa $^{1}$ (Drumstick) & 12,9 & 12,5 & 12,3 & 12,5 & 12,9 & 5,82 \\
Gordura abdominal (Abdominal fat) & 1,34 & 1,18 & 1,25 & 1,31 & 1,29 & 25,98 \\
\hline
\end{tabular}

${ }^{1}$ Efeito quadrático $(P<0,02)$ (Quadratic effect, $\left.P<0.02\right)$. 
Tabela 5 - Equações de regressão para conversão alimentar (CA), consumo de met + cis $(\mathrm{CMC})$, peso relativo da sobrecoxa (RSOBRE) e peso relativo do pulmão (RPUL) de frangos de corte aos 42 dias de idade mantidas em ambiente termoneutro alimentados com rações com diferentes níveis de metionina + cistina (MC)

Tabela 5 - Regression equations for feed:gain ratio(FG), met + cys intake $(\mathrm{MCl})$, relative weight of drumstick (DRU) and relative weight of lung (RLUN) of male broilers at 42 days of age kept under thermoneutral environment fed diets with different levels of methionine + cystine $(M C)$

\begin{tabular}{lcc}
\hline $\begin{array}{l}\text { Variável } \\
\text { Variable }\end{array}$ & $\begin{array}{l}\text { Regressão } \\
\text { Regression }\end{array}$ & $* \mathrm{R}^{2}$ ou r \\
\hline CA & $=3,75698-5,18384 \mathrm{MC}+3,56288 \mathrm{MC}^{2}$ & 0,82 \\
$F G$ & $\hat{\mathrm{Y}}=-1,78603+33,0145 \mathrm{MC} * *$ & 0,99 \\
$\begin{array}{l}\text { CMC } \\
M C I\end{array}$ & $\hat{\mathrm{Y}}=53,5788-110,193 \mathrm{MC}+73,5608 \mathrm{MC}^{2}$ & 0,98 \\
$\begin{array}{l}\text { RSOBRE } \\
\text { DRU }\end{array}$ & $\hat{\mathrm{Y}}$ & \\
\hline
\end{tabular}

* $R^{2}$ - equação quadrática; $r^{2}$ - equação linear.

* $R^{2}$-quadratic equation; $r^{2}$ - linear equation.

relação negativa entre os níveis de metionina + cistina da ração e a deposição de gordura abdominal.

A divergência de resultados entre os trabalhos pode estar relacionada, entre outros fatores, à linhagem das aves utilizadas nos estudos.

\section{Conclusões}

O nível de $0,727 \%$ de met + cis total, correspondente ao nível calculado de $0,661 \%$ de met + cis digestível (0,213\% de met + cis/Mcal de EM) e a uma relação met + cis/ lis digestível de $72 \%$, proporcionou melhor conversão alimentar em frangos de corte de 22 a 42 dias de idade mantidos em ambiente termoneutro.

\section{Literatura Citada}

ALBINO, L.F.T.; SILVA, S.H.M.; VARGAS JR., J.G. et al. Níveis de metionina + cistina para frangos de corte de 1 a 21 e 22 a 42 dias de idade. Revista Brasileira de Zootecnia, v.28, p.519525, 1999.

BAKER, D.H.; HAN, Y. Digestible lysine requirement of male and female broiler chicks during the period three to six weeks posthatching. Poultry Science, v.73, p.739-745, 1994.
GOMES, P.C.; ALBINO, L.F.T.; SILVA, M.A. Criação de frangos de corte. Viçosa, MG: Universidade Federal de Viçosa, 1996. 16p. (Informe Técnico, 78).

JENSEN, L.S.; FANCHER, B.I. Sulfur amino acid requirement of broiler chickens from 3 to 6 weeks of age. Poultry Science, v.68, p.163-168, 1989.

MITCHELL, N.S.; ROBBINS, K.R. Effect of dietary energy level on the total sulfur amino acid of growing broilers. Tenesse farm and Home Science, v.125, p.6-10, 1984.

NATIONAL RESEARCH COUNCIL - NRC. Nutrient requirements of poultry. 9.rev.ed. Washington, D.C.: National Academy Press, 1994. 155p.

RODRIGUEIRO, R.J.B.; ALBINO, L.F.T.; ROSTAGNO, H.S. et al. Exigência de metionina+cistina para frangos de corte na fase de crescimento e abate. Revista Brasileira de Zootecnia, v.29, p.507-517, 2000.

ROSTAGNO, H.S.; ALBINO, L.F.T.; DONZELE, J.L. et al. Tabelas brasileiras para aves e suínos: composição de alimentos e exigências nutricionais. 2.ed. Viçosa, MG: Universidade Federal de Viçosa, 2000. 141p.

SCHEUERMANN, G.N.; BILGILI, S.F.; HESS, J.B. et al. Breast muscle development in commercial broiler chickens. Poultry Science. v.82, p.1648-1658, 2003.

SCHUTTE, J.B.; PACK, M. Sulfur amino acid requirement of broilers chicken from fourteen to thirty-eight days of age. 1. Performance and carcass yield. Poultry Science, v.74, p.480487, 1995a.

SCHUTTE, J.B.; PACK, M. Effects of dietary sulfur-containing amino acids on performance and breast meat deposition of broiler chicks during the growing and finishing phases. Poultry Science, v.74, p.747-762, 1995b.

SCHUTTE, J.B.; JONG, J.; SMINK, W. et al. Replacement value of betaine for DL-methionine in male broiler chicks. Poultry Science, v.76, p.321-325, 1997.

$\hat{Y}$ SEKIS, S.; SCOTT, M.L.; NESHEIM, M.C. The effect of methionine deficiency on body weight, food and energy utilization in the chick. Poultry Science, v.54, p.1184-1188, 1975.

SILVA, D.J.; QUEIROZ, A.C.Análise de alimentos: métodos químicos e biológicos. 3.ed. Viçosa, MG: Universidade Federal de Viçosa, 2002. 235p.

SILVA, M.A.; ALBINO, L.F.T.; ROSTAGNO, H.S. et al. Exigências nutricionais em metionina+cistina para frangos de corte, em função do nível de proteína bruta da ração. Revista Brasileira de Zootecnia, v.26, p.357-363, 1997.

SILVA, R.G.C.; LANA, G.R.Q.; RABELLO, C.B. et al. Exigência de metionina + cistina para frangos de corte machos de 1 a 21 e de 22 a 42 dias de idade, em clima tropical. Revista Brasileira Zootecnia, v.34, p.2399-2407, 2005 (supl.).

UNIVERSIDADE FEDERAL DE VIÇOSA - UFV. SAEG. Central de Processamento de Dados - UFV/CPD. SAEG - Sistema de Análise Estatística e Genética. Viçosa, MG: Universidade Federal de Viçosa, 1992. 59p. 